\section{Absceso prostático tratado mediante resección transuretral de próstata: reporte de un caso}

Carrillo-Córdova LD, Lemus-Mena GR, Rodríguez-Robles J, Vitar-Sandoval J, Hernández-Farías MA, Jaspersen-Álvarez J y colaboradores.

\section{Resumen}

ANTECEDENTES: El absceso prostático es un padecimiento excepcional, cuya incidencia representa $0.5 \%$ de todas las alteraciones prostáticas. Con el desarrollo de antibióticos de amplio espectro ha disminuido su incidencia y complicaciones asociadas.

CASO CLÍNICO: Paciente masculino de 68 años de edad, con antecedente de diabetes tipo 2, de 30 años de evolución; inició con fiebre, náuseas y vómito; dolor punzante en la región perineal, con irradiación escrotal (intensidad 5/10), acompañado de afectación al estado general. A la exploración física, mediante tacto rectal, se palpó la próstata de $4 \times 4 \mathrm{~cm}$, con aumento de la temperatura, ahulada, sin sospecha de malignidad. El ultrasonido mostró la próstata de 3.13 x $2.83 \times 3.77 \mathrm{~cm}$, de $17 \mathrm{~g}$, hipoecogenicidad en su interior, de $3 \times$ $2 \times 2 \mathrm{~cm}$, volumen de $12 \mathrm{cc}$, sugerente de absceso intraprostático. La tomografía simple y contrastada de abdomen y pelvis mostró tres imágenes hipodensas sugerentes de colección intraprostática, de aproximadamente 10 cc cada una. Se estableció el diagnóstico de absceso prostático. El tratamiento consistió en resección transuretral de la próstata. El cultivo del material purulento reportó $E$. coli sensible a carbapenémicos. El esquema de tratamiento se completó con la prescripción del antibiótico.

CONCLUSIÓN: El absceso prostático es una alteración poco frecuente, que puede provocar insuficiencia orgánica múltiple y, en algunos casos, la muerte; por tanto, debe establecerse el diagnóstico oportuno y efectuar la intervención temprana.

PALABRAS CLAVE: Absceso prostático; resección transuretral de próstata; hiperplasia prostática; resección transuretral; sepsis.

Rev Mex Urol. 2018 Jan-Feb;78(1):51-56.

\section{Prostatic abscess treated through transurethral resection of the prostate: $A$ case report}

Carrillo-Córdova LD, Lemus-Mena GR, Rodríguez-Robles J, Vitar-Sandoval J, Hernández-Farías MA, Jaspersen-Álvarez J y colaboradores.

\section{Abstract}

BACKGROUND: Prostatic abscess is an unusual event, with a $0.5 \%$ incidence in relation to all prostatic alterations. Thanks to the devel-
Departamento de urología, Hospital General de México Dr. Eduardo Liceaga, Ciudad de México.

Recibido: abril 2017

Aceptado: noviembre 2017

Correspondencia

Dr. Luis Daniel Carrillo Córdova

carrillocor@gmail.com

Este artículo debe citarse como

Carrillo-Córdova LD, Lemus-Mena GR, RodríguezRobles J, Vitar-Sandoval J, Hernández-Farías MA, Jaspersen-Álvarez J, Moreno-Cabrera JC y col. Absceso prostático tratado mediante resección transuretral de próstata: reporte de un caso. Rev Mex Urol. 2018 ene-feb;78(1):51-56. DOI: https://doi.org/10.24245/revmexurol.v78i1.1401 
opment of broad-spectrum antibiotics, its incidence and associated complications have decreased.

CLINICAL CASE: A 68-year-old man had a past medical history of type 2 diabetes mellitus of 30-year progression. He presented with general malaise, fever, nausea, and vomiting, with stabbing pain in the perineal region, radiating to the scrotum (5/10 intensity). Physical examination with digital rectal examination revealed a warm, rubbery, $4 \times 4-\mathrm{cm}$ prostate, with no suspicion of malignancy. Ultrasound imaging identified a $3.13 \times 2.83 \times 3.77-\mathrm{cm}, 17-\mathrm{g}$, prostate with a hypoechogenic interior of $3 \times 2 \times 2 \mathrm{~cm}$ and a volume of $12 \mathrm{cc}$, suggestive of intraprostatic abscess. Abdominopelvic unenhanced and contrast-enhanced computed tomography revealed three hypodense images, suggestive of an intraprostatic collection, of approximately 10 cc each. Diagnosis was prostatic abscess. Treatment consisted of transurethral resection of the prostate. Culture of the purulent matter reported carbapenem-sensitive $E$. coli. Treatment was completed with that antibiotic.

CONCLUSIONS: Prostatic abscess is an uncommon pathology that can potentially lead to multiple organ failure and death, thus requiring opportune diagnosis and early intervention.

KEYWORDS: Prostatic abscess; Transurethral resection of the prostate; Prostatic hyperplasia; Transurethral resection; Sepsis.

\section{ANTECEDENTES}

El absceso prostático es un padecimiento excepcional, cuya incidencia representa $0.5 \%$ de todas las alteraciones prostáticas. Con el desarrollo de antibióticos de amplio espectro ha disminuido su incidencia y complicaciones asociadas. ${ }^{1}$ Esta alteración suele aparecer entre la quinta y sexta décadas de vida; sin embargo, se ha reportado en pacientes de cualquier edad, incluso en neonatos.

Este padecimiento se origina por el tratamiento inadecuado de una prostatitis bacteriana. En algunos casos la mala reacción al tratamiento o la falta del mismo en un microabsceso inicial puede producir un absceso de diferente dimensión. ${ }^{1,2}$
Los microorganismos más frecuentemente identificados en los abscesos prostáticos son bacilos gramnegativos, sobre todo E. coli, en 60 a $80 \%$ de los casos. Entre otras causas de absceso prostático se encuentra la obstrucción a la salida del flujo, ya sea por hiperplasia prostática, colocación de catéteres uretrales, procedimientos invasivos en la vía urinaria inferior (biopsia prostática), insuficiencia renal crónica, hemodiálisis, enfermedad hepática crónica o infección por VIH. ., Los síntomas prostáticos son variables, desde fiebre, dolor abdominal, disuria, frecuencia urinaria, dolor perineal, incluso tenesmo rectal. A la exploración física puede palparse la próstata reblandecida y aumentada de tamaño, debido a la coexistencia de pus en su interior. 


\section{CASO CLÍNICO}

Paciente masculino de 68 años de edad, con antecedente de diabetes tipo 2, de 30 años de evolución, en tratamiento con insulina NPH, descontrolado y con amputación infracondíleo derecha hacía 4 años, debido a necrobiosis diabética. Quince días antes de ingresar a la unidad hospitalaria inició con fiebre, náuseas y vómito; posteriormente se agregó dolor punzante en la región perineal, con irradiación escrotal (intensidad 5/10), acompañado de afectación al estado general y persistencia de la fiebre, náuseas y vómito. El paciente negó síntomas de vaciamiento y almacenamiento vesical. Inició con disuria, tenesmo y poliuria 24 horas antes de su ingreso. A la exploración física, mediante tacto rectal, se palpó la próstata de $4 \times 4 \mathrm{~cm}$, con aumento de la temperatura, ahulada, sin sospecha de malignidad. El resto del examen resultó sin alteraciones relevantes. Los estudios de laboratorio reportaron: biometría hemática con leucocitos 6.9 , neutrófilos $76 \%$; hemoglobina 11.8 , hematocrito 35.1 y plaquetas 186 ; glucosa 380, urea 95, creatinina 2.2, ácido úrico 7 y Ha1bc 9.8; antígeno prostático específico 2.2; examen general de orina color ámbar, turbio; nitritos negativos, leucocitos incontables, bacterias abundantes, levaduras negativas y eritrocitos de $0-2$ por campo. El ultrasonido mostró la próstata de $3.13 \times 2.83 \times 3.77 \mathrm{~cm}$, de $17 \mathrm{~g}$, hipoecogenicidad en su interior, de $3 \times 2$ $x 2 \mathrm{~cm}$, volumen de $12 \mathrm{cc}$, sugerente de absceso intraprostático (Figura 1). La tomografía simple y contrastada de abdomen y pelvis mostró tres imágenes hipodensas sugerentes de colección intraprostática, de aproximadamente 10 cc cada una (Figura 2). Se efectuaron impregnación antibiótica con imipenem y drenaje quirúrgico de las colecciones intraprostáticas mediante resección transuretral de próstata (Figura 2). El cultivo del material purulento reportó $E$. coli sensible a carbapenémicos. El esquema de tratamiento se completó con la prescripción del antibiótico y se otorgó el alta hospitalaria.

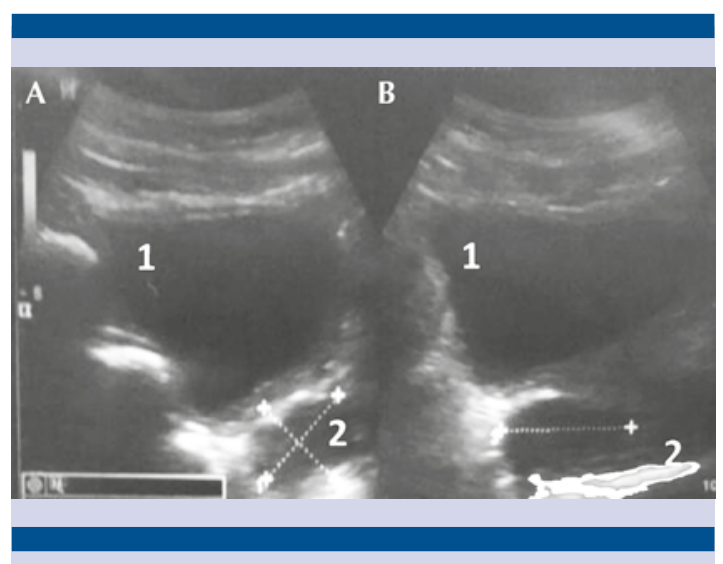

Figura 1. Ultrasonido vesicoprostático abdominal. (A): corte longitudinal que muestra la cavidad vesical [1] y la próstata [2]; (B): corte transversal que evidencia la cavidad vesical [1] y la próstata [2], ambos con imagen hipoecoica en su interior, compatible con un absceso intraprostático de 12 cc.

\section{DISCUSIÓN}

La inmunosupresión y la diabetes mellitus son factores predisponentes de abscesos prostáticos. ${ }^{4}$ El ultrasonido transrectal es el estudio de elección para establecer el diagnóstico de abscesos prostáticos y la mejor técnica a corto plazo; entre sus ventajas, representa un método con exposición a radiaciones bajas y es relativamente económico. $^{5}$

Hasta el momento no se ha establecido ninguna clasificación específica basada en estudios de imagen, ni características clínicas de los diferentes tipos de abscesos prostáticos; sin embargo, se han propuesto diversas clasificaciones, como la de Dell'Atti, quien la realiza con base en las características obtenidas del ultrasonido. ${ }^{6}$ Es importante efectuar el drenaje quirúrgico, incluso en procedimientos de mínima invasión, pero la modalidad terapéutica óptima para cada paciente deberá individualizarse, encaminada a la recuperación rápida y el control de los síntomas. El drenaje de abscesos prostáticos debe realizarse mediante resección transuretral de la 

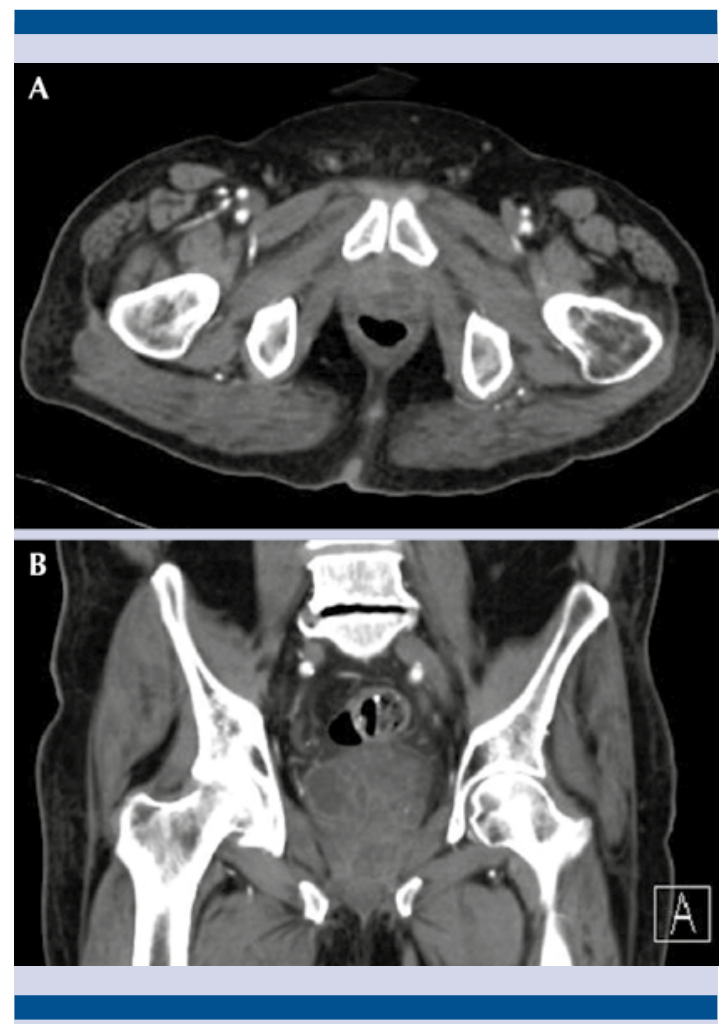

Figura 2. Tomografía computada abdominopélvica en fase simple. (A): corte transversal que muestra la próstata con apariencia heterogénea y pérdida de la anatomía normal. (B): corte coronal donde se observa heterogeneidad intraprostática, con el lóbulo medio prostático que protruye al interior de la vejiga.

próstata, incisión perineal abierta o aspiración transperineal con aguja guiada por ultrasonido transrectal. ${ }^{7-9}$

El patrón de referencia en pacientes con retención aguda de orina, secundaria a prostatitis aguda o absceso prostático, consiste en derivación urinaria por cistostomía suprapúbica, pues la instrumentación uretral puede provocar sepsis..$^{10}$ De estos procedimientos se han realizado múltiples series de casos, una de ellas es la implementación mediante aspiración guiada por ultrasonido transrectal. Por ejemplo, el estudio de Puri y su grupo reportó una efectividad de $100 \%$ y tratamiento de $93 \% .^{11}$
El drenaje transrectal y transperineal se indica en pacientes de mayor edad, en quienes sufren eventos como choque y riesgo de algún procedimiento anestésico elevado. Tanto la resección transuretral de próstata (RTUP), como la incisión transuretral de próstata (ITUP) se indican en pacientes que pueden tolerar el procedimiento y la administración de anestesia general. La en quienes puede retirarse puede efectuarse en pacientes en quienes puede retirarse el absceso residual después de una aspiración primaria o en sujetos ancianos sin contraindicaciones para el procedimiento, incluso en sujetos con hiperplasia prostática obstructiva que genere alteraciones del vaciamiento vesical. Sin embargo, la resección transuretral de la próstata puede incrementar el riesgo de sepsis y bacteriemia. Por tal motivo deben valorarse, de forma preoperatoria, los riesgos y el método más idóneo, según cada caso. ${ }^{12}$

Aunque son poco frecuentes, los abscesos prostáticos pueden drenar hacia el espacio periprostático, la uretra, el recto (fístula rectouretral), el espacio perivesical y periné, incluso hacia el peritoneo y la vejiga, debido al establecimiento del diagnóstico tardío o por drenaje inadecuado. ${ }^{1,13}$

\section{CONCLUSIÓN}

El absceso prostático es una alteración excepcional que puede provocar insuficiencia orgánica múltiple y, en algunos casos, la muerte; por tanto, se requiere establecer el diagnóstico oportuno y efectuar la intervención temprana. En este reporte, el paciente fue tratado mediante resección transuretral de próstata con excelentes resultados y duración corta, sin recurrencia ni complicaciones adicionales.

\section{Conflicto de interés}

Los autores de este trabajo declaran no tener conflicto de interés. 

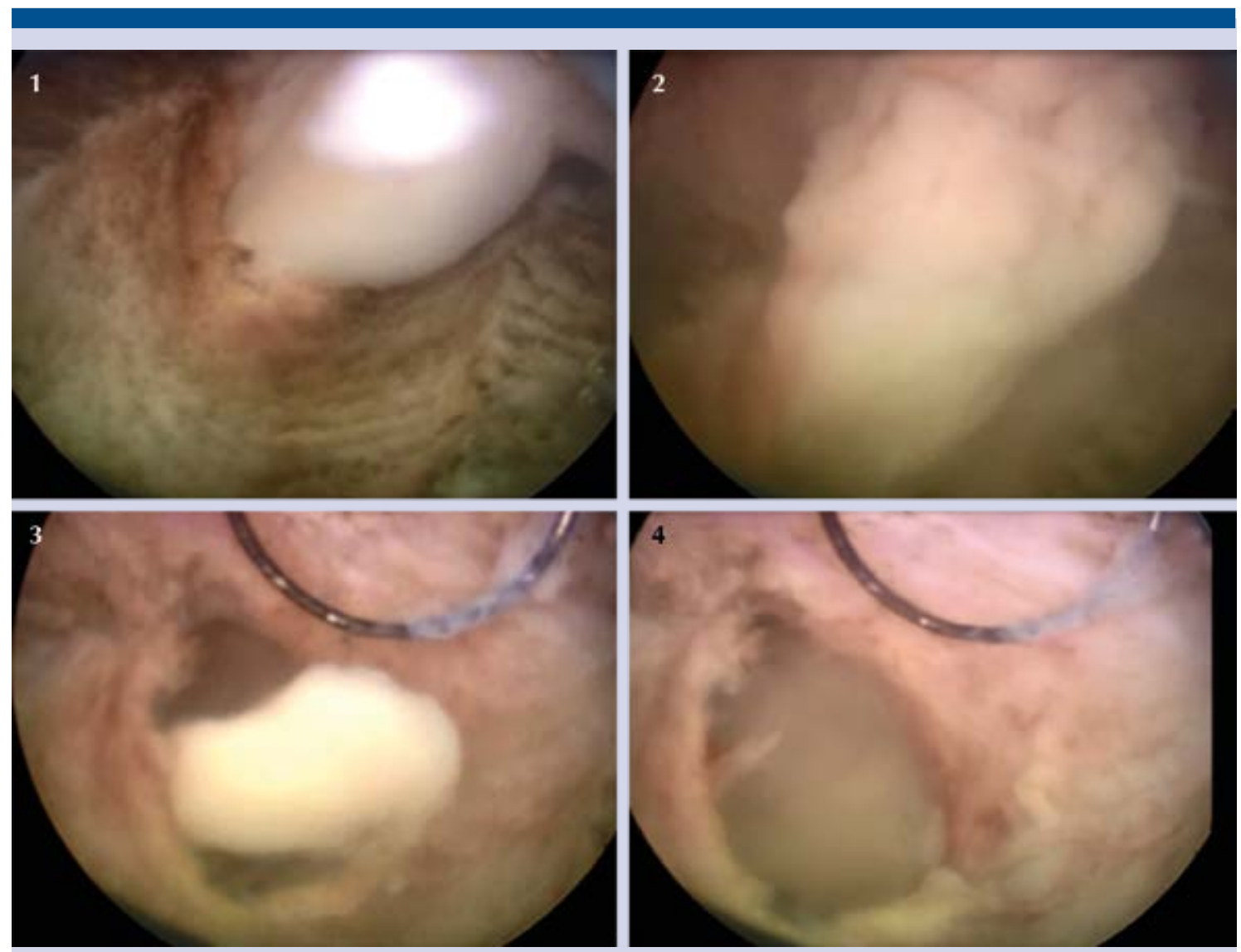

Figura 3. Drenaje del absceso prostático por vía endoscópica transuretral: 1) se realiza destechamiento del absceso, en sentido de las 7:00 h de la carátula del reloj, 2) salida de abundante material purulento (aproximadamente 20 cc), 3) drenaje del absceso mediante compresión del tejido prostático y 4) formación de un pseudodivertículo después de drenar el absceso.

\section{REFERENCIAS}

1. Granados EA, Riley G, Salvador J, Vicente J. Prostatic abscess: diagnosis and treatment. J Urol. 1992;148:80-82.

2. Oliveira $P$, Andrade JA, Porto $\mathrm{HC}$, Filho JE, Vinhaes AF. Diagnosis and treatment of prostatic abscess. Int Braz J Urol. 2003;29:30-34.

3. Langer JE, Cornud F. Inflammatory disorders of the prostate and the distal genital tract. Radiol Clin North Am. 2006;44:665-677.

4. Varkarakis J, Sebe P, Pinggera GM, Bartsch G, Strasser H. Three-dimensional ultrasound guidance for percutaneous drainage of prostatic abscess. Urology. 2004;63:10171020.

5. Bayne $C E$, Davis WA, Rothstein CP, Engel JD. Seminal vesicle abscess following prostate biopsy requiring transgluteal percutaneous drainage. Can J Urol. 2013;20:68116814.

6. Dell'Atti L. A new ultrasound and clinical classification for management of prostatic abscess. Arch Ital Urol Androl 2017;87(3):246-249.

7. Arrabal-Polo MA, Jimenez A, Arrabal-Martin M. Percutaneous drainage of prostatic abscess: case report and literature review. Urol Int 2012;88:118-120. 
8. Collado A, Palou J, Garcia-Penit J, et al. Ultrasoundguided needle aspiration in prostatic abscess. Urology 1999;53(3):548-552

9. Trauzzi SJ, Kay CJ, Kaufman DG, Lowe FC. Management of prostatic abscess in patients with human immunodeficiency syndrome. Urology 1994;43:629-33.

10. Wen SC, Juan YS, Wang CY, et al. Emphysematous prostatic abscess: case series study and review. Int J Infect Dis 2012;16(5):e344-e349.
11. Puri R, Choudhary NS, Kotecha $H$, et al. Endoscopic ultrasound-guided pelvic and prostatic abscess drainage: experience in 30 patients. Indian J Gastroenterol 2014;33(5):410-413.

12. Tai HC. Emphysematous prostatic abscess: a case report and review of literature. J Infect 2007;54(1):e51-e54.

13. Sargent JC, Irwin R. Prostatic abscess: a clinical study of 42 cases. Am J Surg. 1931;11:334-7.

\section{AVISO IMPORTANTE}

La Revista Mexicana de Urología se convierte en una publicación solo digital (www.revistamexicanadeurologia.org.mx) con todas las ventajas que los medios electrónicos ofrecen.

Para consultar el texto completo de los artículos deberá registrarse por una sola vez con su correo electrónico, crear una contraseña, indicar su nombre, apellidos y especialidad.

Esta información es indispensable para saber qué consulta y cuáles son sus intereses, y poder en el futuro inmediato satisfacer sus necesidades de información.

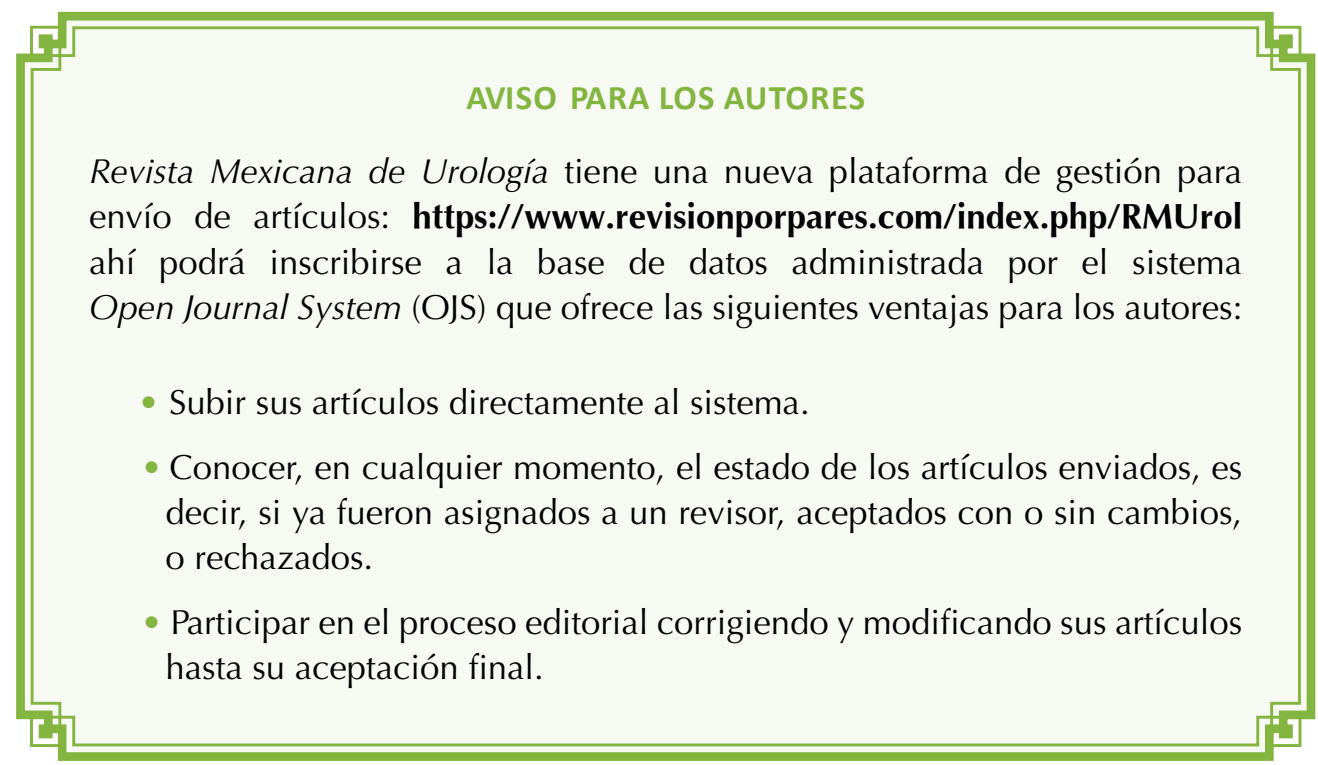

